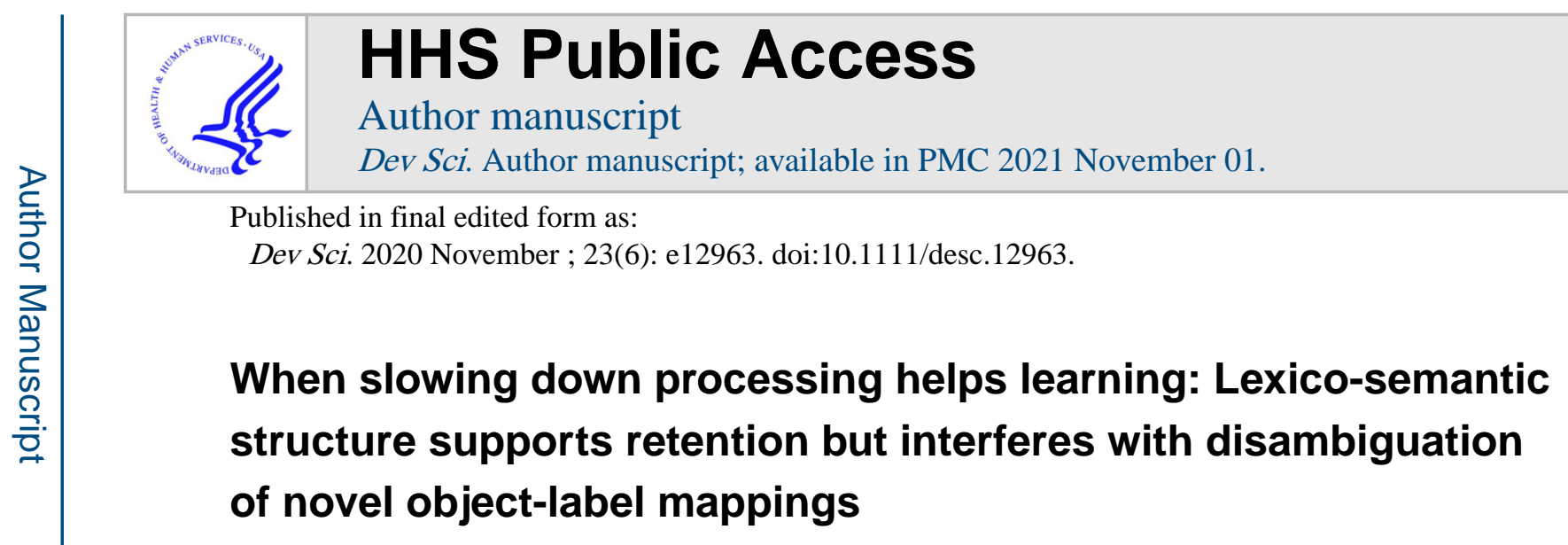

\author{
Arielle Borovsky \\ Department of Speech, Language, and Hearing Sciences, Purdue University, West Lafayette, IN, \\ USA
}

\begin{abstract}
This project explores how children disambiguate and retain novel object-label mappings in the face of semantic similarity. Burgeoning evidence suggests that semantic structure in the developing lexicon promotes word learning in ostensive contexts, while other findings indicate that semantic similarity interferes and temporarily slows familiar word recognition. This project explores how these distinct processes interact when mapping and retaining labels for novel objects (i.e. low-frequency objects that are unfamiliar to toddlers) via disambiguation from a semanticallysimilar familiar referent in 24-month-olds ( $\mathrm{N}=65$ ). Toddlers' log-adjusted looking to labeled target objects (relative to distractor objects) was measured in three conditions: Familiar trials (familiar label spoken while viewing semantically related familiar and novel objects), Disambiguation trials (unfamiliar label spoken while viewing semantically similar familiar and unfamiliar object), and Retention trials (unfamiliar label spoken while viewing novel object pairs). Toddlers' individual vocabulary structure was then compared to performance on each condition. Vocabulary structure was measured at two levels: category-level structure (semantic density) for experimental items, and lexicon-level structure (global clustering coefficient). The findings suggest, consistent with prior results, that semantic density interfered with known word recognition, and facilitated unfamiliar word retention. Children did not show a significant novel word preference during disambiguation, and disambiguation behavior was not impacted by semantic structure. These findings connect seemingly disparate mechanisms of semantic interference in processing and semantic leveraging in word learning. Semantic interference momentarily slows word recognition and resolution of referential uncertainty for novel label-object mappings. Nevertheless, this slowing might support retention by enabling comparison between related objects.
\end{abstract}

\title{
Keywords
}

lexico-semantic development; word learning; disambiguation; semantic networks; eye tracking

Address correspondence to: Arielle Borovsky, Department of Speech, Language, and Hearing Sciences, Purdue University, West Lafayette, IN, USA, 47906, aborovsky@ purdue.edu, Phone: (765) 494-1669, Fax: (765) 496-0771.

Author's note:

I am grateful to the families who participated in this project. Numerous research assistants supported data collection including:

Courtney Yehnert, Kathleen Hopkins, Christopher Cole, Angele Yazbec, and Katherine Chia. This research was financially supported by grants from the National Institutes of Health: DC01368, HD052120. 
How might children's ability to learn words be impacted by their existing knowledge? Emerging research suggests that lexico-semantic structure in the child's existing lexicon is an important piece of this puzzle. Specifically, word learning can be facilitated for novel items that share a dense semantic network of known word meanings, compared to a sparser network (Borovsky, Ellis, Evans, \& Elman, 2016a; Hadley, Dickinson, Hirsh-Pasek, \& Golinkoff, 2019; Hills, Maouene, Maouene, Sheya, \& Smith, 2009; Kaefer, Pinkham, \& Neuman, 2009; Sailor, 2013). Yet little is understood regarding the conditions under which "semantic leveraging" supports learning, especially when learners encounter referential uncertainty and semantic similarity. The current study sheds light on these issues by exploring how lexico-semantic structure in the child's vocabulary and in the learning context affects two commonly studied processes in word learning - referent disambiguation and retention.

Toddlers navigate environments teeming with semantic similarity (Clerkin, Hart, Rehg, Yu, \& Smith, 2017; Sadeghi \& McClelland, 2015), and this environmental structure is reflected in early vocabulary growth (Hills et al., 2009). For example, a toddler eating fruit salad for the first time might encounter a number of (semantically related) fruits - some fruits that they might be able to label (e.g. banana and apple) and others that they might not (e.g. pineapple and kiwi). Toddlers might also vary in their prior experience with fruit, with some knowing many fruits (i.e. having a dense semantic neighborhood), and others knowing only a few (i.e. having a sparser semantic neighborhood). How might toddler's knowledge impact word learning in the face of semantic similarity in the learning environment? Prior research suggests that both semantic similarity in the learning environment and lexico-semantic structure in the child's vocabulary are likely to influence processes in word learning, though this research has largely focused on learning in ostensive contexts, when an unfamiliar referent is clearly identified and named (Borovsky et al., 2016a; Hadley et al., 2019; Neuman, Newman, \& Dwyer, 2011; Storkel \& Adlof, 2009). However, a great deal of word learning does not involve such unambiguous mappings among labels and their objects (Tomasello \& Barton, 1994). Instead, the early learner's world is brimming with items whose labels are both known and unknown. A major theoretical enterprise within developmental science has focused on how children navigate this referential uncertainty, citing a plethora of heuristics and cues that support disambiguation (i.e. appropriately mapping novel objects to novel labels) and retention of novel object-label pairings, (Bloom, 2000; Clerkin et al., 2017; Hollich, Hirsh-Pasek, \& Golinkoff, 2000; Smith \& Yu, 2008). Few studies have explored how lexico-semantic structure supports disambiguation and retention of novel words. A prior study that explored how vocabulary structure influences disambiguation and retention focused on differences in vocabulary composition for items organized by shape (e.g. ball, cup; Perry, Axelsson, \& Horst, 2016). Here, 24-month-olds with larger shape-related vocabularies were better able to retain novel object features and labels, yet this study did not report on how vocabulary structure associated with children's disambiguation performance. Correspondingly, the current project advances prior work by focusing on whether and how semantic structure in the child's own vocabulary influences toddlers' ability to retain novel object-label mappings by resolving referential uncertainty (i.e. disambiguating) among semantically similar novel and known words. 
There is vast literature that explores how children learn to map word labels and their referents. Though young learners have many available cues to word meanings, there is also logical uncertainty in how labels map to potential meanings (Quine, 1960). One way to reduce this referential uncertainty is through the use of a disambiguation strategy (i.e. mutual exclusivity) that preferentially associates novel labels with a novel objects (Markman \& Wachtel, 1988; Merriman, Bowman, \& Macwhinney, 1989). While there is some disagreement surrounding whether children robustly exhibit disambiguation behaviors before the age of two, numerous reports suggest that referent disambiguation and retention skills improve across toddlerhood (Bion, Borovsky, \& Fernald, 2013; Lewis, Cristiano, Lake, Kwan, \& Frank, 2019). For example, Bion and colleagues (2013) found that 18month-old toddlers neither disambiguate nor retain novel objects-label mappings, 24-montholds engaged in disambiguation, but not retention, while 30-month-olds did both. Similarly, a recent meta-analysis of disambiguation effects supports the idea that disambiguation and retention skills become more robust with age (Lewis et al., 2019).

Individual variability in disambiguation and retention behaviors has also been tied to toddlers' lexical knowledge. For example, toddlers with larger vocabularies also show better performance on lexical disambiguation and retention tasks (Bion et al., 2013; Graham, Poulin-Dubois, \& Baker, 1998; Lewis et al., 2019; Mervis \& Bertrand, 1995), suggesting that word retention abilities (indexed by vocabulary size) and disambiguation behaviors go hand-in-hand. McMurray, Horst and Samuelson (2012) propose one potential mechanism by which vocabulary knowledge may support disambiguation. Specifically, they suggest that increased familiarity with known objects in turn facilitates the child's ability to reject a match between a novel label and a known object and instead prioritize the novel label-object mapping (Kucker, McMurray, \& Samuelson, 2015; McMurray, Horst, \& Samuelson, 2012). Similarly, Grassman, Schulze and Tomasello (2015) build on this work by comparing novel referent selection behaviors (i.e. disambiguation) with familiar distractors that are either in the child's receptive vocabulary (understood-only), or are both known and produced (understood+produced) by the child. They find that children show better novel referent selection performance understood+produced words vs. understood-only words, suggesting that increased familiarity with the known words in the understood+produced condition facilitated disambiguation behavior.

This project reconsiders lexical disambiguation and retention phenomena through the lens of two distinct mechanisms that could alternatively enhance or interfere with label-object mapping: semantic leveraging and semantic interference. Semantic leveraging accounts suggest that well-connected lexico-semantic structure promotes word learning (Beckage, Smith, \& Hills, 2011; Borovsky et al., 2016a; Sailor, 2013). Specifically, these accounts posit that word learning is facilitated for novel words in more densely (vs. more sparsely) populated lexico-semantic neighborhoods. For example, a recent study by Borovsky and colleagues (2016a) found improved retention of ostenstively trained novel words from denser semantic categories. In other words, a child who had a dense "vehicle" vocabulary neighborhood and a sparse "animal" vocabulary neighborhood was better able to retain an ostensively trained label-object pairing for novel vehicles vs. animals. 
Semantic interference is a second mechanism that is likely to contribute to word retention and disambiguation in this study. Here, lexico-semantic knowledge can interfere or slow down the recognition of known items due to co-activation of related features and concepts. For instance, a recent study by Vales and Fisher (2019) found that labeled items that appear with a semantically-related competitor object are slower to be selected by 3- to 9-year-olds. This pattern has been echoed in eye-tracking work in infants, toddlers and adults that finds less robust fixations towards items named in semantically related vs. unrelated contexts (Bergelson \& Aslin, 2017; Borovsky, Ellis, Evans, \& Elman, 2016b; Borovsky \& Peters, 2019; Huettig \& Altmann, 2005; Yee \& Sedivy, 2006).

Thus, semantic leveraging and interference mechanisms can have opposing but impactful influences on word recognition, disambiguation and retention and thereby provide interesting theoretical tension and opportunity to enrich our current understanding of disambiguation and retention processes in word learning. On one hand, semantic leveraging accounts suggest that disambiguation and retention processes may be supported by enhanced lexico-semantic structure that improves overall familiarity of known object-label mappings and facilitates connections between known and novel semantically-related items (Borovsky et al., 2016a; Hadley et al., 2019; Kucker, McMurray, \& Samuelson, 2018; McMurray, Horst, \& Samuelson, 2012; Perry, Axelsson, \& Horst, 2016; Sailor, 2013). On the other hand, semantic interference accounts posit that denser semantic structure is likely to interfere with word disambiguation and retention via the co-activation of related features and concepts (Bergelson \& Swingley, 2012; Borovsky \& Peters, 2019; Vales \& Fisher, 2019). These alternating accounts are explored in the current study by measuring how semantic structure impacts novel object-label disambiguation and retention in 24-month-old toddlers. Twenty-four months is an ideal age to explore these questions because, as reviewed above, children of this age show robust disambiguation effects that are also affected by overall vocabulary size, and prior work (using the same novel objects adopted in this study) reports that semantic density influences retention of ostensively-trained novel word labels at this age (Borovsky et al., 2016a). The next sections outline the approach to developing novel stimuli and measuring vocabulary structure before outlining potential study outcomes.

\section{Novel objects in the study}

As this project considers how young children acquire novel words that are semantically related to known items, "novel" items were defined as low-frequency items from several early-learned categories (e.g. draisine: a type of vehicle; mamey: a kind of fruit). To ensure the selected novel labels were unfamiliar, parental report was used to verify that these words were unknown to all toddlers in the study. Although the category membership of these lowfrequency "novel" items was not explicitly mentioned during the study, the novel items were selected to share many visual features in common with other category members (e.g. draisine is made of metal and has gears and wheels, mamey is red-orange, appears juicy with seeds and a skin). Novel items in this project were selected from a prior study that explored the impact of semantic category density on word learning in ostensive contexts (Borovsky et al., 2016a). 


\section{Measuring vocabulary structure}

A key goal of this project is to explore how vocabulary structure in the toddler's lexicon supports disambiguation and retention of novel label-object mappings. Following prior studies that explored the impact of semantic structure on known word processing (Borovsky \& Peters, 2019), vocabulary structure was measured in two ways: at the category-level and lexicon-level. Category level density (i.e. semantic density) was measured as the relative proportion of items in a particular category reported to be produced by a child on a standardized vocabulary checklist (Borovsky et al, 2016a for initial demonstration of this procedure), and reflects the relative density of nearby semantic neighbors. Lexicon-level density was measured using global clustering coefficient (GCC) - a network science metric of average semantic overlap between words and their neighbors across the child's productive vocabulary, thus representing the relative density of near and far neighbors. Higher global clustering coefficient values are argued to support efficient word processing and activation in mature lexicons (Steyvers \& Tenenbaum, 2005). Together, these two measures allow further exploration of how local (category density) and global (GCC) semantic connectivity support processes in disambiguation and retention.

\section{The current study}

The current study measured disambiguation and retention using eye-tracking measures of toddlers' gaze preference for a target object relative a distractor. In disambiguation trials, toddlers gaze towards a novel target and familiar distractor object was measured in response to a novel word label. Retention was measured in subsequent trials where previously labeled novel objects pairs reappeared, while one was labeled. Importantly, prior studies using a very similar trial structure with 24 month-olds have found that performance on disambiguation trials related to individual differences in vocabulary size at 24 months (Bion et al., 2013). This project explored how the child's own vocabulary structure interacts with disambiguation and retention behaviors, using indices of vocabulary structure (semantic category density and GCC, described above). Therefore, this project builds on these prior contributions to explore how mechanisms of semantic leveraging and semantic interference contribute to disambiguation and retention behaviors in 24-month-olds. As prior research suggested that retention of ostensively-taught novel items is supported by denser semantic structure (Borovsky et al., 2016), it was expected that higher category density and GCC would result in better retention of novel items. However, mechanisms of semantic leveraging and interference yield alternating possible outcomes on the disambiguation task. If, as suggested by semantic interference accounts, word recognition is slowed in semantically related contexts, then it is expected that toddlers would show poor disambiguation when named novel objects shared semantic overlap with the familiar distractors, particularly when semantic density or GCC was high. If semantic leveraging supports disambiguation, then it is expected that denser semantic structure would improve performance on disambiguation trials. 


\section{Method}

\section{Participants.}

97 families with 24-month-old toddlers were invited to visit the lab. Of this sample, 11 children did not contribute eye-tracking data due to either the child's refusal to complete the task that day, or experimenter/equipment error. An additional 9 toddlers were excluded from data analysis as they did not meet inclusionary/exclusionary criteria ( 7 were receiving occupational therapy or speech therapy, 2 were hearing a language other than English $>1 \mathrm{hr}$ a day). An additional 12 children attempted or completed the task, but were removed from analysis due to excessive fussiness or trackloss, meaning their final dataset did not include at least two trials in each condition. This left a final dataset consisting of 65 toddlers ( $37 \mathrm{M}, 28$ F, Age range: $725-785$ days, $\mathrm{M}_{\mathrm{age}}=744$ days, $\mathrm{SD}_{\mathrm{age}}=11.99$ days). The remaining sample was all reported to be typically developing and learning primarily English at home. All caregivers provided informed consent to take part in the study, and the study conformed to ethical standards, as reviewed and approved by the Florida State University Institutional Review Board.

\section{Experimental stimuli.}

Selection of category domains and novel items.-Children saw novel and familiar items. Novel items were the same six novel items used in Borovsky and colleagues (2016a). These novel objects were low-frequency items that belonged to six early-acquired categories in which 24-month-olds typically understand several words including: ANIMALS, CLOTHING, VEHICLES, BODY-PARTS and two food subcategories: FRUITS and DRINKS. The visual depictions of these low-frequency items were selected so as to share many of the same visual features of other members in the category. For example, the novel clothing item "Banyan" is made of cloth, appears to fit on a human body, and has clasps and other typical clothing adornments. Novel items are illustrated in Figure 1. Familiar items were selected to be known by a majority of 24-month-olds, according to the CLEX database (Jørgensen, Dale, Bleses, \& Fenson, 2010). Novel and familiar items in each category are listed in Appendix A.

Visual Stimuli.-Each novel and known word in the experiment was represented by a photorealistic representation of the novel item and placed on a $400 \times 400$ pixel image on a white background. In addition to the experimental familiar and novel objects, other known objects were also presented as filler stimuli, as were several additional smaller, colorful cartoon-like images to help maintain and direct the child's attention towards the screen.

Auditory stimuli.-All auditory stimuli were recorded by a female native English speaker in a child-directed voice on a mono channel at $44.1 \mathrm{kHz}$ sampling rate. All novel and familiar labels were normalized to a mean duration and standard intensity using Praat (Boersma \& Weenink, 2012). The speaker also recorded other encouraging phrases that were designed to maintain and capture toddlers' interest. These included tag phrases that followed each experimental item, like "Great job," or "That's cool!" that served to help maintain child attention as well as other encouraging phrases during filler and break trials like "This is fun, let's see some more pictures!” 


\section{Procedure.}

Vocabulary assessment.-To gain an overall measure of each child's productive vocabulary size and structure, we asked each parent to complete the MacArthur-Bates Communicative Development Inventory: Words \& Sentences (MBCDI; Fenson et al., 2007). Percentile scores on this assessment ranged from $1^{\text {st }}-99^{\text {th }}$ percentile, and children were reported to produce between 16-662 words (Figure 2; $\mathrm{M}_{\% \text { tile }}=51.5 ; \mathrm{SD} \%$ tile $=26.2 ; \mathrm{M}_{\mathrm{words}}=$ 300.5; Median $_{\text {words }}=268 ; \mathrm{SD}_{\text {words }}=158.5$ )

Child experimental item knowledge.-Each child's caregiver was asked to rate their child's knowledge of known and novel items in the experiment on a scale from 1 (child does not understand the word) to 4 (child definitely understands the word). Trials were removed from the experiment where caregivers marked less than a ' 2 ' for familiar target items and marked " 4 " for novel items.

Experimental procedure.-After the caregiver had completed vocabulary and background questionnaires, the caregiver and child moved to an adjacent room to begin testing. The child was seated in a car seat in front of a 17" computer monitor (1024 x 724 pixel resolution) with an SR Research Eyelink 1000+ eye-tracking camera mounted underneath. The caregiver and an experimenter were seated on either side of the child. Another experimenter remained out of view behind a curtain and controlled the experimental presentation and eye-tracking equipment. Caregivers were asked to refrain from naming images that appeared during the experiment.

The eye-tracker was focused and calibrated using a standard five-point procedure before the experimental trials began. Figure 3 depicts the sequence of events within a trial. Each trial was initiated once the child fixated to a small, colorful image ( $30 \times 30$ pixels) at the center of the monitor. This central image then disappeared, and the target and distractor image appeared in silence for $2000 \mathrm{~ms}$ to allow the toddler to preview the object images and locations. Next, a gaze-contingent central image (100 x 100 pixels) appeared along with a pre-recorded attention-getting verbal stimulus (“Look!"), while the target and distractor items remained on the screen. This central stimulus served to ensure that the child was attending to the experiment at the onset of the spoken label. Once the child fixated to this gaze-contingent central stimulus, it disappeared, leaving only the target and distractor image, and the target label was spoken (e.g. "Car!") followed by an encouraging tag message (e.g. "Great job!"). Target and distractor images remained on the screen for $4000 \mathrm{~ms}$ after the onset of the spoken label. All image presentations were counterbalanced so that they appeared equally as a target and distractor, and on the left and right side of the screen, yielding a balanced within-subjects design.

All toddlers saw 36 experimental trials comprising 12 trials in each of three conditions: (1) Familiar word trials, (2) Disambiguation trials, and (3) Retention trials (Figure 4). Familiar word trials included a (labeled) familiar target and novel distractor object. Disambiguation trials included a (labeled) novel target and a familiar distractor. Retention trials included pairs of novel objects that had been previously labeled during the Disambiguation trials. As in Bion and colleagues (2013), Disambiguation and Familiar word trials preceded Retention 
trials. In addition to the experimental trials, children saw additional filler trials (24 total) containing pairs of familiar objects interspersed with the retention trials to help toddlers maintain interest during the relatively more difficult retention trial portion of the study. Trials were presented across three blocks of 12 experimental trials, with eight filler-trials in each block, and children were allowed to take longer breaks between blocks, if needed. The entire procedure lasted approximately 10 minutes.

Eye-tracking recording apparatus and method.-Using an SR-Research Eyelink $1000+$ eye-tracker, we recorded eye-movements at $500 \mathrm{~Hz}$ from a single eye. These data were binned into $50 \mathrm{~ms}$ time intervals for plotting and analysis. We defined spatial areas of interest for analysis as the two 400 x 400 pixel squares in which the Target and Distractor images appeared.

Analytic approach.-We calculated several metrics of vocabulary size and structure. Productive vocabulary size was calculated using the toddler's MBCDI report (Fenson et al., 2007). We then calculated two metrics of vocabulary structure, described below:

Category-level structure (Category density).-Semantic category density was calculated following procedures in several prior studies (Borovsky et al., 2016a, 2016b; Borovsky \& Peters, 2019). Using the items in each child's MBCDI report, the total proportion of words spoken in the (six) semantic categories comprising the experimental materials was calculated. Each child's proportion of words spoken in each category was then rank-ordered, and each child's top ranked (3) categories were assigned to a High density condition, and each child's three lowest-ranked categories were assigned to a Low density condition. The proportion of participants in High and Low density categories is reported in Table 1.

Whole-lexicon structure (Global clustering coefficient).-A graph-theoretic approach was used to index each child's lexico-semantic inter-connectivity via global clustering coefficient (GCC). The nodes in each child's network represented the nouns that they were reported to say on the MBCDI. Following established procedures (Peters \& Borovsky, 2019), words (nodes) were linked when they shared at least two perceptual and/or functional semantic features, according to a combined set of feature production norms for all nouns in the MBCDI (Peters, McRae, \& Borovsky, in prep.). GCC is calculated as the total number of words that are connected in closed triangles divided by the total number of connected triples (Newman, Moore, \& Watts, 2000). A connected triple is any set of three words (nodes) that shares semantic links (e.g. spoon-soup, spoon-fork), and a triple is defined as "closed" if all three nodes are interconnected (e.g. cow-goat, goat-horse, horsecow). GCC values range from 0 to 1 ; a 0 value denotes that the child's lexicon has no connected triples, and 1 indicates that all triplets are closed. Thus, unlike semantic category density, this measure captures an index of global structure in the lexicon beyond pre-defined category membership.

Eye-movement data analysis plan.-Based on prior research, it was expected that performance should be improved for items from denser categories, and for children with larger and more highly interconnected lexicons. Therefore, separate analyses were carried 
out on each condition (Familiar, Disambiguation, and Retention trials). In each condition, analyses sought to measure the contribution of vocabulary size (as median split vocabulary group) and structure (Category Density and GCC) on performance by first plotting the timecourse of looking, and then through statistical analyses that measure looking behavior in a coarser broad time window analysis, followed by a finer time-grained analysis. Each analytic step is described below.

Timecourse visualization.-Eye-movement patterns were initially visualized in each experimental condition using log-gaze timecourse plots in Figure 5. These log-gaze plots illustrate the total proportion of fixations to target relative distractor, to highlight time points where looks to the target exceeded that of the distractor, as denoted by positive values. Following previously established procedure for eye-movement analyses in a similar previous study (Borovsky et al. 2016a), log-gaze proportions were calculated as the log-proportion of fixations to the Target image divided by fixations to the Distractor [ $\log$ (Proportion looking to Target/Proportion looking to Distractor) at each $50 \mathrm{~ms}$ time bin. Because log values are undefined at 0 , if proportion looking to the Target or Distractor (numerator or denominator in the formula) was zero, then a small value (.01) was added to the proportion. Positive loggaze proportion values indicate a target preference, while negative values denote a distractor preference. This log-ratio procedure has been adopted in favor of using raw proportion values in a number of eye-movement studies with a variety of populations (e.g. Arai, van Gompel, \& Scheepers, 2007; Borovsky et al., 2016a; Knoeferle \& Kreysa, 2012; Wienholz $\&$ Lieberman, 2019). This log-gaze approach presents two statistical advantages. First, because looks to the Target and Distractor are not linearly independent (e.g. more looks to the Target means fewer to the Distractor and vice-versa) a ratio expresses the bias to view one object relative to the other, which does not violate statistical assumptions of linear independence. Second, raw proportion ratios are fixed between 0 and 1, which violates homogeneity of variance assumptions, whereas log-gaze ratios can vary, in principle, between positive and negative infinity, thereby yielding distributions that are more appropriate for parametric statistical testing (see Arai et al. 2007 for additional discussion of statistical advantages).

Analysis of eye-movement patterns over broad time window.-Next, statistical analyses of eye-movement accuracy patterns in each condition over a pre-defined time window were conducted. Following precedent from a similar study of disambiguation and retention (Bion et al., 2013), the analysis focused on an identical time window of analysis (300 to $3300 \mathrm{~ms}$ post-label onset) over which mean log-gaze accuracy was calculated. The period of analysis is started at $300 \mathrm{~ms}$ after label onset to account for the delay it takes for a young child to program an eye-movement in response to a linguistic stimulus (Fernald, Zangl, Portillo, \& Marchman, 2008).

Finer-grained analysis of eye-movement patterns.-Pre-defined mean-accuracy windows that collapse across a large window of time (in our case, $3000 \mathrm{~ms}$ ) can mask more fleeting dynamics of lexical recognition in eye-movement data. To identify time windows where effects occur in time-series data while controlling for multiple comparisons, a nonparametric cluster-based bootstrapping approach that can identify time windows over which 
eye-movements differ from chance or another condition was implemented (Delle Luche, Durrant, Poltrock, \& Floccia, 2015; Groppe, Urbach, \& Kutas, 2011; Maris \& Oostenveld, 2007; Von Holzen \& Mani, 2012). This procedure (described in detail in Maris \& Oostenveld, 2007) is carried out in several steps. The first step initially identifies temporally adjacent time bins that significantly differ from a pre-specified threshold, and sums the tstatistics of these adjacent time windows to create a "cluster-t statistic." To determine whether this cluster $t$ value exceeds chance with a family-wise error rate $<.05$, this cluster- $t$ value is compared against a cluster-t distribution generated via 1000 random assortments of the compared data to calculate Monte-Carlo $p$-value. Therefore, this procedure supports inferences about the timing of significant differences across conditions without requiring a pre-determined time window, as in the broad time window analyses. This cluster-based permutation approach was used to carry out two types of analyses in each condition. The first analysis sought to identify when target fixations exceeded those to the distractor as a function of category density and GCC. The second analysis measured at which time points higher and lower semantic category density and GCC influenced looking behavior.

Eye-movement data cleaning and trackloss removal.-The initial dataset comprised 2340 trials with 67 toddlers. Several data cleaning steps were carried out to arrive at a final dataset for analysis. First, parental report was used to verify that the familiar words in the study were known to the children, resulting in the removal of 3 trials (.13\% of trials) in familiar word condition, leaving 2337 trials. Then, to ensure that all novel items in the study were not previously known by the children, trials were removed for novel items that the parents indicated were familiar to the child, resulting in the removal of 8 additional trials (.34\% of trials), leaving 2329 trials. Next, following prior precedent in studies of known and novel word processing (Borovsky et al., 2016a; Borovsky \& Peters, 2019) trials were removed in which toddlers did not view the target or distractor image, or the eye-tracker failed to record data for at least $20 \%$ of the analysis period ( 300 to $3300 \mathrm{~ms}$ ). This standard identified 157 trials (6.74\%) for trackloss removal, leaving 2172 trials. After trackloss removal, two additional children were removed from the sample, as they did not contribute at least 2 trials in each condition, leaving a total of 65 participants and 2108 trials that contributed to the statistical analysis (710 Familiar word trials, 702 Disambiguation trials, and 696 Retention trials). The dataset and analytic code are available at https://osf.io/tqycs/.

\section{Results}

\section{Visualizing timecourse of eye-movements in Familiar, Disambiguation and Retention Trials.}

Toddlers' timecourse of fixations were initially plotted across each experimental condition to inspect timing and impact of semantic category structure (Figure 5a), and as a function of overall lexical-connectivity, indexed by global clustering coefficient in Figure 5b. Several key patterns are evident in this timecourse figure, including that children quickly recognized familiar objects in the FN trials, as illustrated by a target preference in the log-gaze plots, and that there appeared to be differences as a function of semantic category structure in the familiar (FN) and retention (NN) trials. These patterns are explored in subsequent statistical analyses below. 


\section{Broad time windows accuracy analysis in Familiar, Disambiguation and Retention trials.}

The goal of this analysis was to measure the interaction of semantic structure and vocabulary size on target word recognition in each of the experimental conditions separately. As described in detail in the analytic approach, broad time window accuracy for each trial was defined as the log-proportion of fixations to the Target relative Distractor from 300 to 3,000 ms after the onset of the spoken word. Linear-mixed effects regression (LMER) using the lme4 library in R, version 3.5.3 (Bates, Maechler, Bolker, \& Walker, 2015; R Core Team, 2019) was used to analyze the impact of vocabulary size and structure on each condition, while controlling for random effects. To control for potential problems with collinearity and to simplify interpretation of the statistical results, each fixed effect factor was centered, and categorical variables (category density) were sum coded (High $=1$, Low $=-1$ ), and continuous variables were standardized. Initial models sought to include both participants and items as random effects in the analyses; however, not all of these models converged in all conditions. Instead, all models converged when items only were included as a random effect. Therefore, to standardize the statistical approach across conditions, only items was included as a random effect. Full model results for each experimental condition is reported in Table 2-Table 4. The formula which was applied to the statistical analysis in all conditions, including fixed and random effects is represented below:

$$
\text { LogGaze } \sim(\text { GCC }+ \text { Category Density }) * \text { Vocabulary Size }+(1 \backslash \text { Items })
$$

A positive, significant intercept in familiar word (FN) trials (Table 2) indicated that, as expected, toddlers' log-fixations to the familiar words exceeded zero, indicating that 24month-olds recognized familiar items when they were labeled by looking more towards the target relative the distractor image. In contrast, for trials involving novel word recognition (disambiguation and retention) the intercept did not exceed zero, indicating that looks to the novel target objects did not exceed distractor looks across the broad time window in these conditions. The remaining statistical output illustrated in Tables 2-4 indicates that, over these broad time bins, there were no significant effects as a function of vocabulary size, semantic category density, or global cluster coefficient in any condition.

\section{Fine-grained accuracy analysis in Familiar, Disambiguation and Retention trials.}

Broad time windows of analysis in eye-tracking can average over and mask dynamic effects that occur over shorter time periods. Therefore, cluster-based permutation analyses were used to identify sequential periods of time (i.e. clusters) where looks to the target exceed looks to the distractor while controlling for family-wise error rate due to multiple comparison (see analytic approach above for fuller description). The first analysis, summarized in Table 5, sought to identify time periods where looks to the target significantly exceeded looks to the distractor across experimental conditions and by vocabulary structure. As expected, for Familiar trials, the cluster analysis identified large time windows spanning most of the trial period over which toddlers showed a target preference (see Familiar Trials in Table 5). These large time clusters are evident in both high and low density conditions, indicating that toddlers recognized the familiar words in the study irrespective of the semantic structure surrounding the word. In contrast, during the 
Disambiguation trials, the cluster analyses did not identify any period of time in which children preferred the target, suggesting a pattern where children looked back-and-forth among target and distractor across the trial and did not correctly map the novel label to the novel object. In contrast, toddlers exhibited a target preference between 1800-2450 ms after label onset in the high semantic category density Retention trials. This difference in performance between the Disambiguation and Retention trials indicates that, despite the toddlers' failure to appropriately map the novel label to its referent during the Disambiguation trials, toddlers successfully retained the appropriate mapping in the high semantic category density condition only.

The goal of the next analyses was to determine whether and when vocabulary structure differentially impacted performance in each experimental condition. A nonparametric cluster analysis approach compared the log-gaze of looks between higher and lower semantic category density, and higher and lower global clustering coefficient in each experimental condition separately. Table 6 illustrates the results of these analyses. The results indicate that toddlers looked more towards the low density Familiar words vs. higher density Familiar words from 1300-1450 ms after label onset. This finding indicates that, familiar items in lower-density categories experience less semantic interference from a semantically related distractor than those in higher density categories. There were no differences as a function of semantic structure in the Disambiguation condition at any time point. This lack of difference is likely driven by the pattern in the previous analysis which illustrated a lack of Target preference across all semantic structure conditions in the Disambiguation trials. Despite the lack of semantic structure effects in the Disambiguation trials, the results of the Retention analysis indicated that toddlers retained words more effectively in higher (vs. lower) density categories from 2100-2300 ms, suggesting that higher semantic density supported learning of novel items, consistent with semantic leveraging accounts.

\section{Discussion}

While prior research had prioritized the importance of building a large vocabulary in toddlerhood (Lee, 2011; Marchman \& Bates, 1994), an emerging area of research has begun to explore the impacts of variability in the semantic structure within the child's lexicon (Beckage et al., 2011; Colunga \& Sims, 2017; Stella, Beckage, \& Brede, 2017). Recent advances in network science have yielded exciting findings that suggest that the course of vocabulary development is shaped by processes that help children learn words that share similarity with their existing vocabulary and environment (Hills et al., 2009; Hills, Maouene, Riordan, \& Smith, 2010). Relatedly, the child's understanding of familiar items in a learning context can support their ability to navigate referential uncertainty (Grassmann, Schulze, \& Tomasello, 2015; McMurray et al., 2012), suggesting that the child's knowledge in the immediate learning context can support learning, in addition to overall vocabulary size.

A separate emerging research strand on the development of lexical processing skills had suggested that word recognition may be temporarily slowed by the child's recognition of semantic relations among words (Bergelson \& Aslin, 2017; Vales \& Fisher, 2019). With these seemingly discordant phenomena in mind, this project sought to connect these ideas by measuring how lexico-structure in the child's productive vocabulary impacts their ability to 
retain novel object-label mappings via an important strategy in word learning - that of disambiguation - when they are faced with semantically related objects.

Two alternative accounts were considered to be consistent with these divergent phenomena. The first account (semantic leveraging) posits that density in semantic structure should support learning, while the other (semantic interference) suggests that such density in semantic structure should interfere when children are navigating referential uncertainty in a semantically related context.

The current findings pave a way to link both accounts, and help to illuminate how and why semantic structure might simultaneously slow word recognition and enhance learning. These connections are supported by three important patterns in the data. The first two patterns replicate prior findings in this area. First, object recognition in semantically related contexts was slower for denser vs. sparser semantic categories, consistent with prior reports that semantically related distractors slow word recognition in children (Bergelson \& Aslin, 2017; Borovsky \& Peters, 2019; Vales \& Fisher, 2019). Secondly, novel object retention was boosted in denser vs. sparser categories, also extending prior findings for learning in ostensive contexts (Borovsky et al., 2016a), and dovetailing reports in preschool and schoolage contexts that building semantic networks via storybook instruction supports further vocabulary growth (Hadley et al., 2019; Kaefer et al., 2009).

While these first two outcomes may seem at odds, the third major finding suggests a way to connect them. In the disambiguation condition there was not robust evidence for successful mapping of the novel label to the referent, in contrast to other studies exploring this phenomenon at this age (Bion, Borovsky, \& Fernald, 2013; Horst \& Samuelson, 2008). Specifically, the fixation patterns in the disambiguation condition, averaged across the broad time window, indicate no preference for the familiar or novel item, and inspection of the time course data indicates perhaps more fleeting preferences for the familiar item, as suggested by negative log-gaze values for some portions of the trial. This failure to disambiguate can be attributed to a novel aspect of this study's design, where familiar and novel objects share semantic similarity. This semantic relation between the novel target and familiar distractor contrasts with prior studies exploring mutually-exclusive referent selection in toddlers. Specifically, prior disambiguation studies have typically sought to avoid semantic overlap between familiar and novel items by using unusual objects to guarantee their novelty (Horst \& Hout, 2016; Horst, Samuelson, Kucker, \& McMurray, 2011). In contrast, the stimuli in this study were specifically selected to share many perceptual features in common with other objects in their intended semantic category. For example, the novel VEHICLE, draisine, had wheels, and metal parts that clearly indicated its membership in a vehicle category, while the fruit item, MAMEY, had seeds, skin, and a color consistent with many other fruits (falling along the red-orange spectrum; Figure 1).

Thus, semantic similarity in the disambiguation trials seems to have led children to consider (either explicitly or implicitly) the semantic connection between the familiar and novel object pairs, leading to what, at first glance, appeared to be a missed opportunity to learn the appropriate mapping in a failure to preferentially gaze towards labeled novel object. However, this back-and-forth viewing pattern between the novel and familiar object during 
disambiguation trials had consequences for retention, such that increased density boosted retention performance. Thus, while the semantic relation between objects interfered with processing in the moment during the disambiguation trials, this semantic overlap boosted the child's ability to recognize correspondences between other similar category members that supported later retention of items in higher density categories. This effect of density on retention is consistent with findings from recent modeling work, which suggest that words that share many perceptual connections with other known words are earlier acquired (Hills, Maouene, Maouene, Sheya, \& Smith, 2009; Peters \& Borovsky, 2019)

Finally, it is important to note that while there were alternating effects of category density on recognition and retention, neither of these processes were impacted by a measure of overall lexico-semantic network connectivity, global clustering coefficient. This outcome suggests that semantic structure impacts word recognition and retention via the local neighborhood surrounding a word, and not by connectivity from distant neighbors in the child's lexicon. The pattern of slowed familiar word recognition for high density neighborhoods mirrors findings in the adult eye-tracking literature (Chen \& Mirman, 2012; Mirman \& Magnuson, 2009), where near semantic neighbors (akin to high category density in this study) slows word recognition. Importantly, these parallels to the adult literature indicate that adult-like psycholinguistic mechanisms of semantic activation start to emerge even in toddlerhood. Further, the observation that category density but not lexicon-level density supported retention suggests that this semantic leveraging effect on learning is boosted by near neighbors, and less impacted by the more complex, larger "metropolis" of words that comprises the child's lexicon. As suggested by one reviewer, it is also possible that disambiguation and other cognitive abilities may grow in tandem, supporting child's ability to attend to, select, and retain the appropriate referent. Given these parallels, further research would be warranted to distinguish whether and to what degree non-verbal cognitive skills support disambiguation and retention.

This project also illuminates an important distinction between the impact of semantic structure on lexical processing and retention. Here, dense structure supported word retention, but interfered with word recognition. Thus, while prior accounts that link processing and learning posit that speedy processing supports learning (Christiansen \& Chater, 2016; Fernald, Perfors, \& Marchman, 2006; Hurtado, Marchman, \& Fernald, 2008; McMurray, Horst, \& Samuelson, 2012; McMurray et al., 2012), the current findings suggest that slowing down processing during referential uncertainty might support the child's ability to scaffold their prior knowledge during novel object-label mappings. These differential effects of semantic structure over varying time scales adds weight to other theoretical accounts that consider the different timescales over which real-time familiar word recognition processes and novel learning act (McMurray, 2016). Namely, while word recognition unfolds rapidly over a period of milliseconds, word learning and retention processes unfold over much longer timescales involving multiple interactions and opportunities for reinforcement and elaboration of lexical knowledge. Thus, in a word learning context, transient initial interference from known semantic competitors might serve to slow down processing in the first moments when a word is encountered, but may ultimately serve to enhance retention and learning over the long run. Future studies are needed to explore how semantic structure 
supports learning and recognition as novel words are learned and become familiar over time (Horst, 2013).

In sum, the current findings add to a burgeoning literature that refines our understanding of how and why building a large and well-connected vocabulary matters. Building lexicosemantic structure in vocabulary might support children's subsequent language growth because it helps children recognize correspondences between word meanings by slowing down lexical recognition in the face of semantic similarity.

\section{Supplementary Material}

Refer to Web version on PubMed Central for supplementary material.

\section{Data availability statement:}

The dataset and analytic code that support the findings of this study are available at: https:// osf.io/tqyes/

\section{References}

Arai M, van Gompel RPG, \& Scheepers C (2007). Priming ditransitive structures in comprehension. Cognitive Psychology, 54(3), 218-250. 10.1016/j.cogpsych.2006.07.001 [PubMed: 16973144]

Bates D, Maechler M, Bolker B, \& Walker S (2015). Fitting Linear Mixed-Effects Models Using lme4. Journal Of Statistical Software, 67(1), 1-48. 10.18637/jss.v067.i01

Beckage N, Smith L, \& Hills T (2011). Small worlds and semantic network growth in typical and late talkers. PLoS ONE, 6(5), e19348. 10.1371/journal.pone.0019348 [PubMed: 21589924]

Bergelson E, \& Aslin RN (2017). Nature and origins of the lexicon in 6-mo-olds. Proceedings of the National Academy of Sciences of the United States of America, 114(49), 12916-12921. 10.1073/ pnas.1712966114 [PubMed: 29158399]

Bergelson E, \& Swingley D (2012). At 6-9 months, human infants know the meanings of many common nouns. Proceedings of the National Academy of Sciences, 109(9), 3253-3258.

Bion RAH, Borovsky A, \& Fernald A (2013). Fast mapping, slow learning: Disambiguation of novel word-object mappings in relation to vocabulary learning at 18,24 , and 30months. Cognition, 126(1). 10.1016/j.cognition.2012.08.008

Bloom P (2000). How Children Learn the Meanings of Words. Cambridge, MA: MIT Press. Retrieved from https://mitpress.mit.edu/books/how-children-learn-meanings-words

Boersma P, \& Weenink D (2012). Praat: doing phonetics by computer. Retrieved from http:// www.praat.org/

Borovsky A, Ellis EMEM, Evans JL, \& Elman JLJL (2016a). Lexical leverage: Category knowledge boosts real-time novel word recognition in two-year-olds. Developmental Science, 19(6), 918-932. 10.1111/desc.12343 [PubMed: 26452444]

Borovsky A, Ellis EM, Evans JL, \& Elman JL (2016b). Semantic structure in vocabulary knowledge interacts with lexical and sentence processing in infancy. Child Development, 87(6), 1893-1908. 10.1111/cdev.12554 [PubMed: 27302575]

Borovsky A, \& Peters RE (2019). Vocabulary size and structure affects real-time lexical recognition in 18-month-olds. PLOS ONE, 14(7), e0219290. 10.1371/journal.pone.0219290 [PubMed: 31295282]

Chen Q, \& Mirman D (2012). Competition and cooperation among similar representations: Toward a unified account of facilitative and inhibitory effects of lexical neighbors. Psychological Review, 119(2), 417-430. 10.1037/a0027175 [PubMed: 22352357]

Christiansen MH, \& Chater N (2016). The Now-or-Never bottleneck: A fundamental constraint on language. Behavioral and Brain Sciences, 39, e62. 10.1017/S0140525X1500031X 
Clerkin EM, Hart E, Rehg JM, Yu C, \& Smith LB (2017). Real-world visual statistics and infants' first-learned object names. Philosophical Transactions of the Royal Society B: Biological Sciences, 372(1711). 10.1098/rstb.2016.0055

Colunga E, \& Sims CE (2017). Not Only Size Matters: Early-Talker and Late-Talker Vocabularies Support Different Word-Learning Biases in Babies and Networks. Cognitive Science, 41, 73-95. 10.1111/cogs.12409 [PubMed: 27873349]

Delle Luche C, Durrant S, Poltrock S, \& Floccia C (2015). A methodological investigation of the Intermodal Preferential Looking paradigm: Methods of analyses, picture selection and data rejection criteria. Infant Behavior and Development, 40, 151-172. 10.1016/j.infbeh.2015.05.005 [PubMed: 26176183]

Fenson L, Marchman VA, Thal DJ, Dale PS, Reznick JS, \& Bates E (2007). MacArthur-Bates Communicative Development Inventories: User's Guide and Technical Manual, Second Edition. Baltimore, MD: Brookes Publishing.

Fernald A, Perfors A, \& Marchman VA (2006). Picking up speed in understanding: Speech processing efficiency and vocabulary growth across the 2nd year. Developmental Psychology, 42(1), 98-116. 10.1037/0012-1649.42.1.98 [PubMed: 16420121]

Fernald A, Zangl R, Portillo AL, \& Marchman VA (2008). Looking while listening: Using eye movements to monitor spoken language comprehension by infants and young children. In Sekerina I, Fernandez E, \& Clahsen H (Eds.), Language Processing in Children. Amsterdam: Benjamins.

Graham SA, Poulin-Dubois D, \& Baker RK (1998). Infants' disambiguation of novel object words. First Language, 18(53), 149-164. 10.1177/014272379801805302

Grassmann S, Schulze C, \& Tomasello M (2015). Children's level of word knowledge predicts their exclusion of familiar objects as referents of novel words. Frontiers in Psychology, 6, 1200. 10.3389/fpsyg.2015.01200 [PubMed: 26322005]

Groppe DM, Urbach TP, \& Kutas M (2011). Mass univariate analysis of event-related brain potentials/ fields I: a critical tutorial review. Psychophysiology, 48(12), 1711-1725. 10.1111/ j.1469-8986.2011.01273.x [PubMed: 21895683]

Hadley EB, Dickinson DK, Hirsh-Pasek K, \& Golinkoff RM (2019). Building Semantic Networks: The Impact of a Vocabulary Intervention on Preschoolers' Depth of Word Knowledge. Reading Research Quarterly, 54(1), 41-61. 10.1002/rrq.225

Hills TT, Maouene M, Maouene J, Sheya A, \& Smith LB (2009). Longitudinal analysis of early semantic networks: Preferential attachment or preferential acquisition? Psychological Science, 20(6), 729-739. 10.1111/j.1467-9280.2009.02365.x [PubMed: 19470123]

Hills Thomas T, Maouene J, Riordan B, \& Smith LB (2010). The associative structure of language: Contextual diversity in early word learning. Journal Of Memory And Language, 63(3), 259-273. [PubMed: 20835374]

Hills TT, Maouene M, Maouene J, Sheya A, \& Smith L (2009). Categorical structure among shared features in networks of early-learned nouns. Cognition, 112(3), 381-396. 10.1016/ j.cognition.2009.06.002 [PubMed: 19576579]

Hollich GJ, Hirsh-Pasek KA, \& Golinkoff RM (2000). Breaking the language barrier: an ermegentist coalistion model for the origins of word learning. Monographs of the Society for Research in Child Development, 65(3, Serial No. 262).

Horst JS, \& Samuelson LK (2008). Fast Mapping but Poor Retention by 24-Month-Old Infants. Infancy, 13(2), 128-157. 10.1080/15250000701795598 [PubMed: 33412722]

Horst Jessica S. (2013). Context and repetition in word learning. Frontiers in Psychology, 4, 149. 10.3389/fpsyg.2013.00149 [PubMed: 23580347]

Horst Jessica S., \& Hout MC (2016). The Novel Object and Unusual Name (NOUN) Database: A collection of novel images for use in experimental research. Behavior Research Methods, 48(4), 1393-1409. 10.3758/s13428-015-0647-3 [PubMed: 26424438]

Horst Jessica S., Samuelson LK, Kucker SC, \& McMurray B (2011). What's new? Children prefer novelty in referent selection. Cognition, 118(2), 234-244. 10.1016/J.COGNITION.2010.10.015 [PubMed: 21092945]

Huettig F, \& Altmann G (2005). Word meaning and the control of eye fixation: Semantic competitor effects and the visual world paradigm. Cognition, 96(1), B23-B32. [PubMed: 15833303] 
Hurtado N, Marchman VA, \& Fernald A (2008). Does input influence uptake? Links between maternal talk, processing speed and vocabulary size in Spanish-learning children. Developmental Science, 11(6), F31-F39. 10.1111/j.1467-7687.2008.00768.x [PubMed: 19046145]

Jørgensen RN, Dale PS, Bleses D, \& Fenson L (2010). CLEX: A cross-linguistic lexical norms database. Journal of Child Language, 37(2), 419-428. 10.1017/S0305000909009544 [PubMed: 19570318]

Kaefer T, Pinkham AM, \& Neuman SB (2009). Taxonomic Organization Scaffolds Young Children's Learning from Storybooks: A Design Experiment. Society for Research on Educational Effectiveness.

Knoeferle P, \& Kreysa H (2012). Can speaker gaze modulate syntactic structuring and thematic role assignment during spoken sentence comprehension? Frontiers in Psychology, 3. 10.3389/ fpsyg.2012.00538

Kucker SC, McMurray B, \& Samuelson LK (2015). Slowing Down Fast Mapping: Redefining the Dynamics of Word Learning. Child Development Perspectives, 9(2), 74-78. 10.1111/cdep.12110 [PubMed: 26918026]

Kucker SC, McMurray B, \& Samuelson LK (2018). Too Much of a Good Thing: How Novelty Biases and Vocabulary Influence Known and Novel Referent Selection in 18-Month-Old Children and Associative Learning Models. Cognitive Science, 42 Suppl 2(Suppl 2), 463-493. 10.1111/ cogs.12610 [PubMed: 29630722]

Lee J (2011). Size matters: Early vocabulary as a predictor of language and literacy competence. Applied Psycholinguistics, 32(1), 69-92. 10.1017/S0142716410000299

Lewis M, Cristiano V, Lake BM, Kwan T, \& Frank MC (2019). The role of developmental change and linguistic experience in the mutual exclusivity effect. PsyArXiv Preprint, (2013). 10.31234/osf.io/ wsx3a

Marchman VA, \& Bates E (1994). Continuity in lexical and morphological development: a test of the critical mass hypothesis. Journal of Child Language, 21(02), 339-366. 10.1017/ S0305000900009302 [PubMed: 7929685]

Maris E, \& Oostenveld R (2007). Nonparametric statistical testing of EEG- and MEG-data. Journal of Neuroscience Methods, 164(1), 177-190. 10.1016/j.jneumeth.2007.03.024 [PubMed: 17517438]

Markman EM, \& Wachtel GF (1988). Children's use of mutual exclusivity to constrain the meanings of words. Cognitive Psychology, 20(2), 121-157. 10.1016/0010-0285(88)90017-5 [PubMed: 3365937]

McMurray Bob. (2016). Language at Three Timescales: The Role of Real-Time Processes in Language Development and Evolution. Topics in Cognitive Science, 8(2), 393-407. 10.1111/tops.12201 [PubMed: 26991438]

McMurray Bob, Horst JSS, \& Samuelson LKK (2012). Word learning emerges from the interaction of online referent selection and slow associative learning. Psychological Review, 119(4), 831. 10.1037/a0029872 [PubMed: 23088341]

Merriman WE, Bowman LL, \& Macwhinney B (1989). The mutual exclusivity bias in children's word learning. Monographs of the Society for Research in Child Development, 54(3), 1-129.

Mervis CB, \& Bertrand J (1995). Acquisition of the novel name-nameless category (N3C) principle by young children who have Down syndrome. American Journal on Mental Retardation, 100(3), 231243. [PubMed: 8554770]

Mirman D, \& Magnuson JS (2009). Dynamics of activation of semantically similar concepts during spoken word recognition. Memory \& Cognition, 37(7), 1026-1039. 10.3758/MC.37.7.1026 [PubMed: 19744941]

Neuman S, Newman EH, \& Dwyer J (2011). Educational effects of a vocabulary intervention on preschoolers word knowledge and conceptual development: A cluster-randomized trial. Reading Research Quarterly, 46(3), 249-272. 10.1598/RRQ.46.3.3

Newman MEJ, Moore C, \& Watts DJ (2000). Mean-Field Solution of the Small-World Network Model. Physical Review Letters, 84(14), 3201-3204. 10.1103/PhysRevLett.84.3201 [PubMed: 11019047] 
Perry LK, Axelsson EL, \& Horst JS (2016). Learning What to Remember: Vocabulary Knowledge and Children's Memory for Object Names and Features. Infant and Child Development, 25(4), 247258. 10.1002/icd.1933

Peters RE, \& Borovsky A (2019). Modeling early lexico-semantic network development: Perceptual features matter most. Journal of Experimental Psychology: General, 148(4), 763-782. 10.1037/ xge0000596 [PubMed: 30973265]

Peters RE, McRae K, \& Borovsky A (in prep.). Semantic feature norms for early-acquired nouns.

Quine W. v O. (1960). Word and object MIT press. Cambridge, MA.

R Core Team. (2019). R: A language and environment for statistical computing. Vienna, Austria: R Foundation for Statistical Computing.

Sadeghi Z, \& McClelland JL (2015). You shall know an object by the company it keeps: An investigation of semantic representations derived from object co-occurrence in visual scenes. Neuropsychologia, 76, 52-61. 10.1016/j.neuropsychologia.2014.08.031 [PubMed: 25196838]

Sailor KM (2013). Is vocabulary growth influenced by the relations among words in a language learner's vocabulary? Journal of Experimental Psychology: Learning Memory and Cognition, 39(5), 1657-1662. 10.1037/a0032993

Smith L, \& Yu C (2008). Infants rapidly learn word-referent mappings via cross-situational statistics. Cognition, 106(3), 1558-1568. 10.1016/j.cognition.2007.06.010 [PubMed: 17692305]

Stella M, Beckage NM, \& Brede M (2017). Multiplex lexical networks reveal patterns in early word acquisition in children. Scientific Reports, 7, 46730. 10.1038/srep46730 [PubMed: 28436476]

Steyvers M, \& Tenenbaum JB (2005). The large-scale structure of semantic networks: Statistical analyses and a model of semantic growth. Cognitive Science, 29(1), 41-78. 10.1207/ s15516709cog2901_3 [PubMed: 21702767]

Storkel HL, \& Adlof SM (2009). The effect of semantic set size on word learning by preschool children. Journal of Speech, Language, and Hearing Research, 52(2), 306-320.

Tomasello M, \& Barton ME (1994). Learning words in nonostensive contexts. Developmental Psychology, 30(5), 639-650. 10.1037/0012-1649.30.5.639

Vales C, \& Fisher AV (2019). When Stronger Knowledge Slows You Down: Semantic Relatedness Predicts Children's Co-Activation of Related Items in a Visual Search Paradigm. Cognitive Science, 43(6), e12746. 10.1111/cogs.12746 [PubMed: 31204802]

Von Holzen K, \& Mani N (2012). Language nonselective lexical access in bilingual toddlers. J Exp Child Psychol, 113(4), 569-586. 10.1016/j.jecp.2012.08.001 [PubMed: 22980955]

Wienholz A, \& Lieberman AM (2019). Semantic processing of adjectives and nouns in American Sign Language: effects of reference ambiguity and word order across development. Journal of Cultural Cognitive Science, 1-18.

Yee E, \& Sedivy JC (2006). Eye movements to pictures reveal transient semantic activation during spoken word recognition. Journal of Experimental Psychology: Learning, Memory, and Cognition; Journal of Experimental Psychology: Learning, Memory, and Cognition, 32(1), 1-14. 


\section{Research Highlights}

- $\quad$ Study addresses alternating accounts of how lexico-semantic structure in toddlers' emerging lexicon influences disambiguation and retention of novel object-label mappings

- 24-month-olds completed tasks of familiar word recognition, novel word disambiguation and novel word retention.

- Toddlers' lexico-semantic structure interfered with familiar word recognition, but boosted novel word retention.

- $\quad$ Results advance theoretical understanding of how emerging semantic structure interacts with lexical processing, disambiguation and retention. 


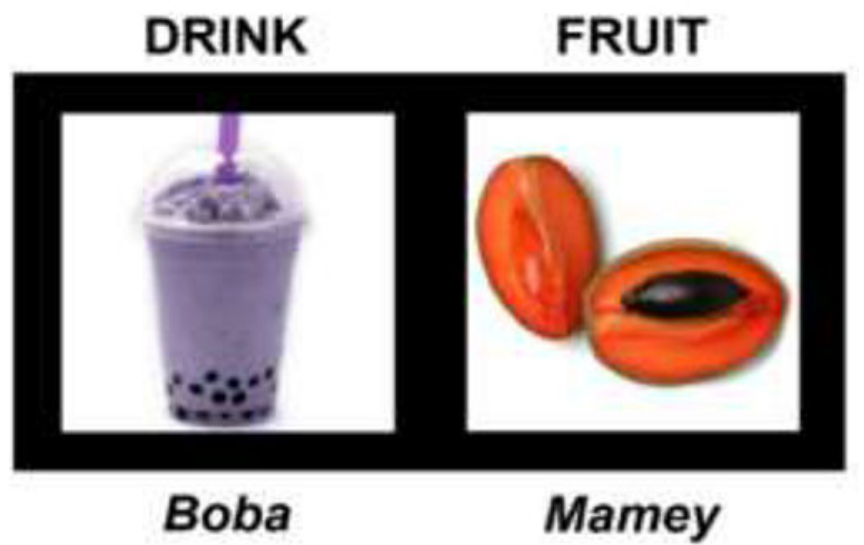

ANIMAL

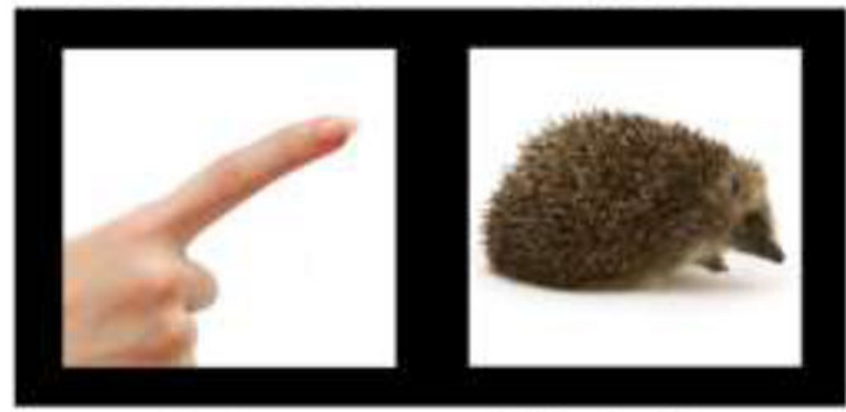

Index

Hedgehog

\section{CLOTHES}

VEHICLES

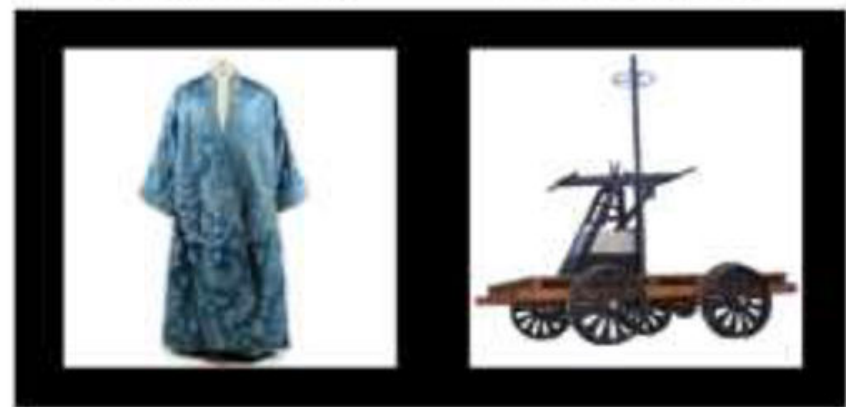

Banyan

Drasine

Figure 1.

Illustration of novel word stimuli and categories used in the study 
MBCDI Productive Vocabulary

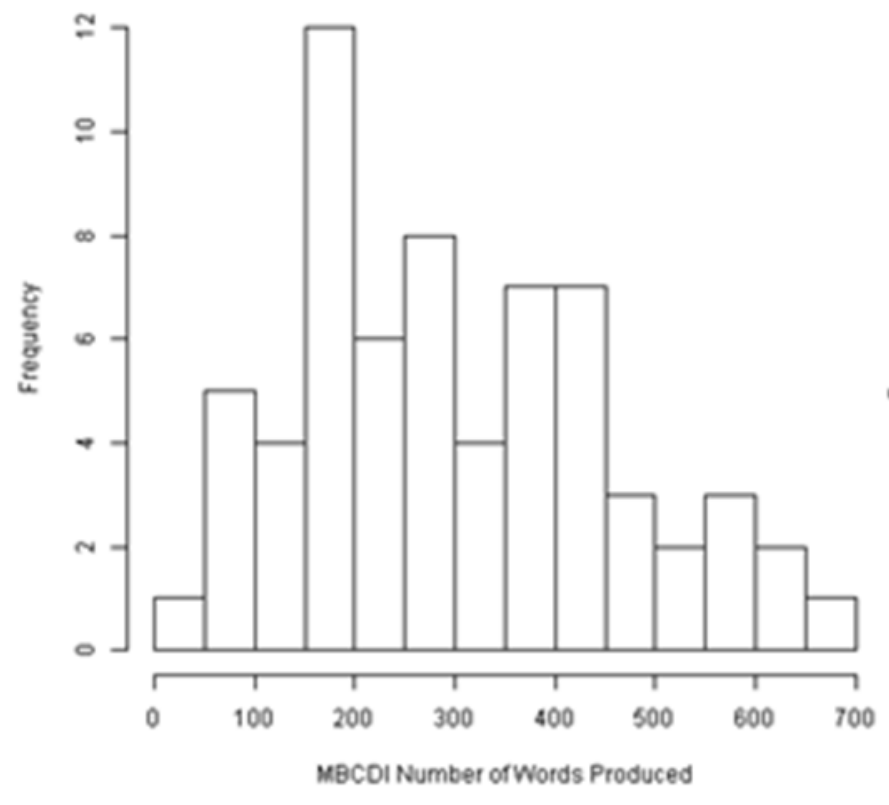

MBCDI Percentile Scores

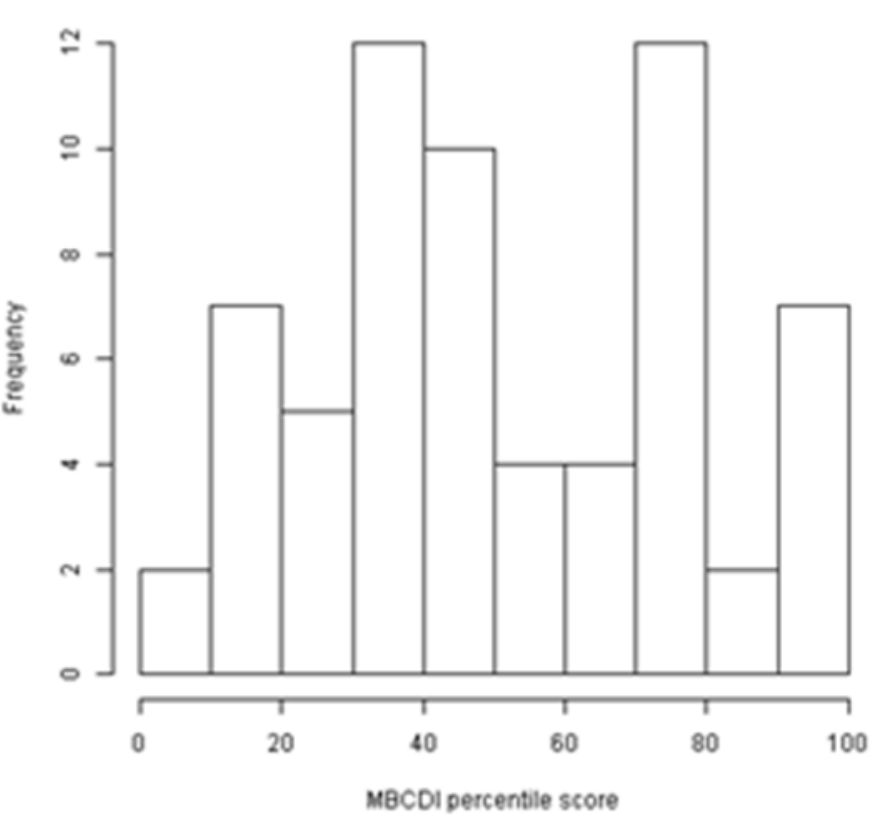

Figure 2.

Histograms of participants' productive vocabulary raw scores and percentiles. 


\section{Trial Structure}

1. Silent preview (2000 ms)

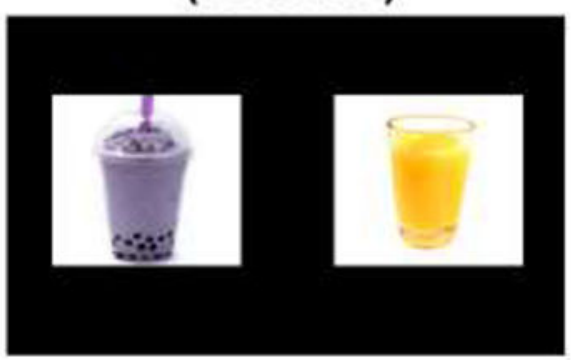

2. Central image appears (gaze contingent timing)

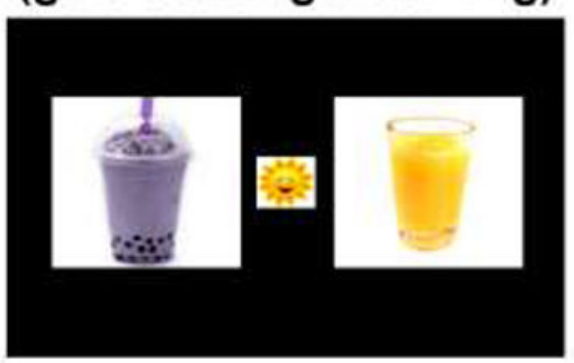

3. Label onset (4000 ms)

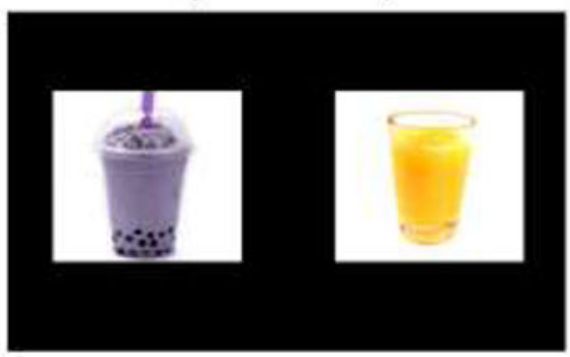

Figure 3.

Illustration of trial event sequence. (1) Trial begins with the appearance of two images on the screen, which appear in silence for $2000 \mathrm{~ms}$ before the (2) appearance of a central stimulus. This central stimulus remains on screen until gaze is registered on the central image for 100 ms. (3) The central stimulus then disappears and the target label is spoken (e.g. Juice! That's cool!). The images remain on the screen for $4000 \mathrm{~ms}$ after the onset of the spoken target label. Note that trial is initiated by gaze towards a central pre-trial attention getter (not pictured here). 
Familiar: Familiar-Novel (FN)

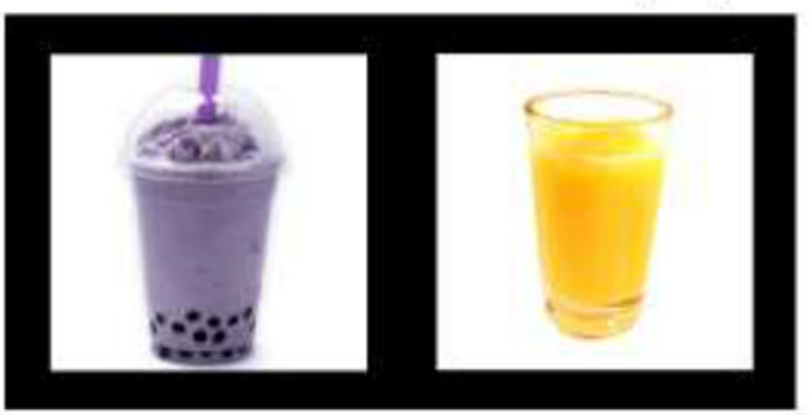

"juice"

\section{Disambiguation: Novel-Familiar (NF)}

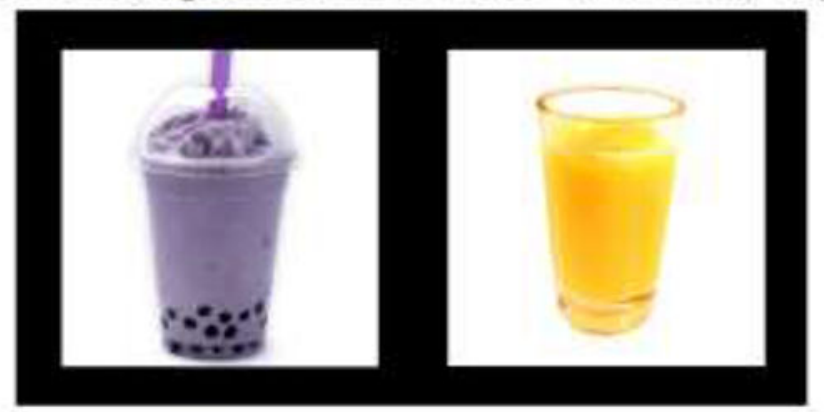

"boba"

\section{Retention: Novel-Novel (NN)}

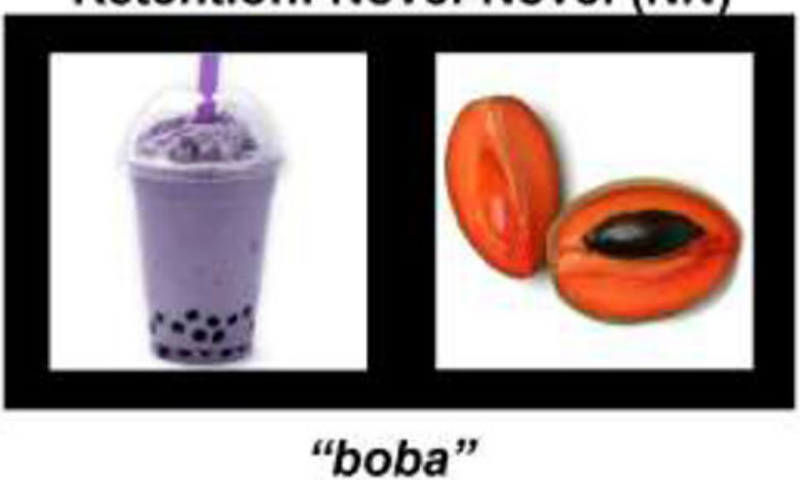

Figure 4.

Illustration of experimental conditions. FN and NF trials consist of familiar and novel images. Familiar trials label the known object, while Disambiguation (NF) trials label the unknown/novel object. Retention of the novel label-object pairing is tested in NN trials, where two previously labeled objects appear, while one is labeled. 


\section{A) $\rightarrow$ Semantic·Category-Density}
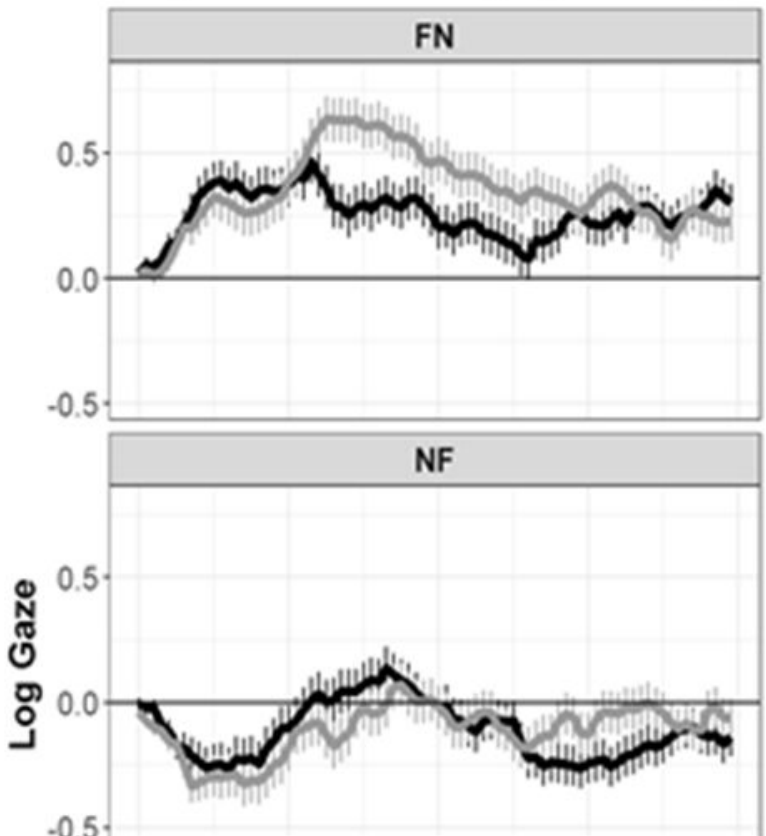

$-0.5$
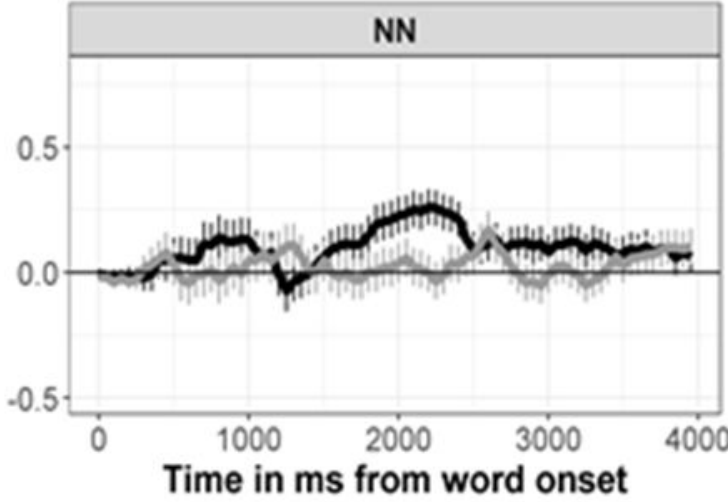

B)-Global-Clustering-Coefficient
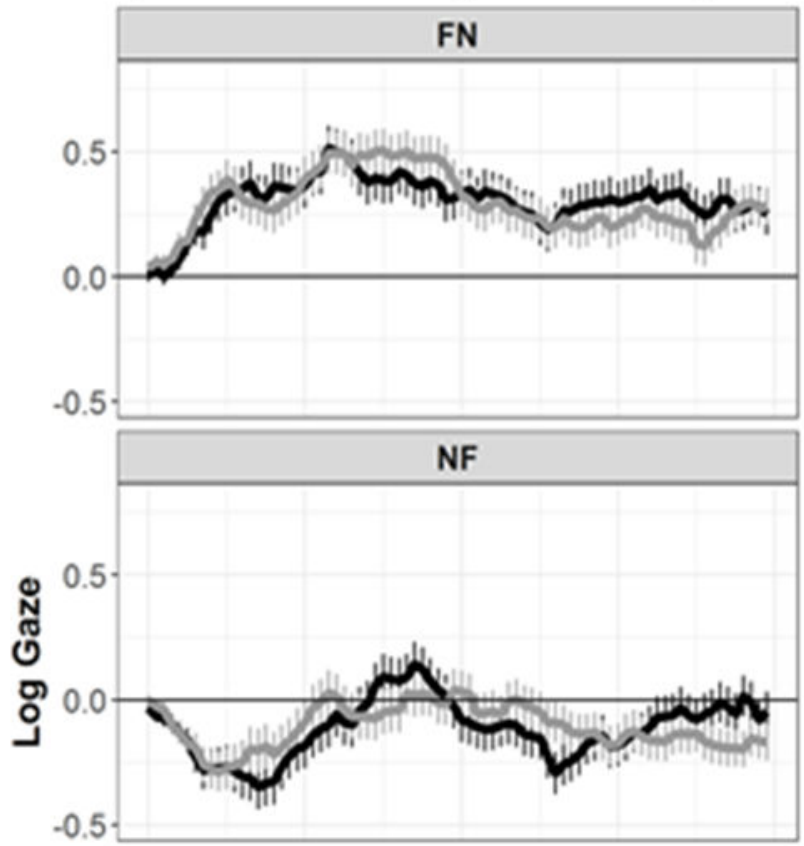

- High

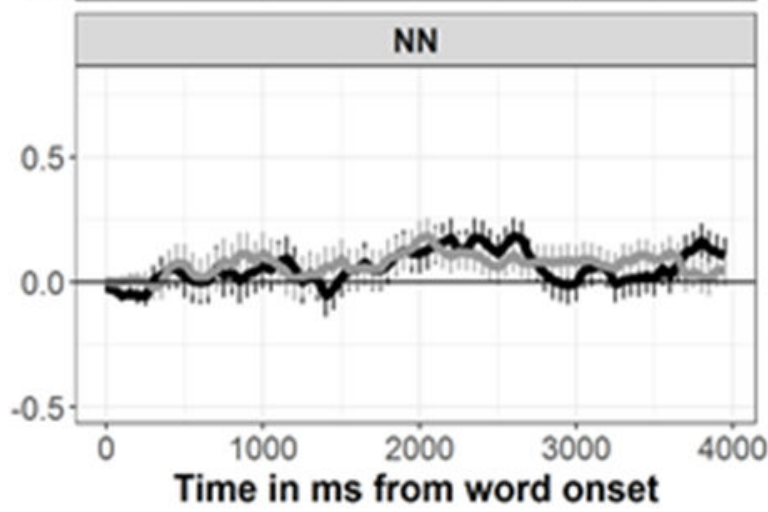

Figure 5.

Time course chart of log fixation proportions to Target / Distractor for Familiar (FN), Disambiguation (NF) and Retention trials (NN) for High and Low (A) Semantic category density, (B) Global clustering coefficient / Lexical connectivity plotted in $50 \mathrm{~ms}$ bins from label onset. Log gaze values greater than 0 indicate a Target preference, and less than 0 denote a Distractor preference 
Table 1.

Proportion of participants with high or low semantic category density in experiment categories

\begin{tabular}{|c|c|c|}
\hline Category & $\underline{\text { High }}$ & Low \\
\hline ANIMALS & .54 & .46 \\
\hline BODY-PARTS & .66 & .34 \\
\hline CLOTHING & .09 & .91 \\
\hline DRINK & .55 & .45 \\
\hline FRUIT & .71 & .29 \\
\hline VEHICLES & .54 & .46 \\
\hline
\end{tabular}


Table 2.

LMER analysis of accuracy for Familiar trials from 300-3300 ms

\begin{tabular}{lllll}
\hline & Estimate & Std. Error & t-value & p-value \\
(Intercept) & $\mathbf{0 . 3 0}$ & $\mathbf{0 . 0 8}$ & $\mathbf{3 . 6 5}$ & $\mathbf{. 0 0 3}$ \\
Vocabulary size & -0.022 & 0.04 & -0.55 & .58 \\
Semantic category density & -0.028 & 0.04 & -0.76 & .45 \\
Global Cluster Coefficient & -0.10 & 0.08 & -1.23 & .22 \\
Voc. x Sem. Cat. Density & 0.036 & 0.04 & 1.03 & .30 \\
Voc. x GCC & -0.04 & 0.05 & -.70 & .49 \\
Correlation of fixed effects*** & & & & \\
\hline & Intercept & 2 & & 3 \\
1. Vocabulary size & .086 & .008 & & .495 \\
2. Semantic category density & -.002 & -- & & -.004 \\
3. Global Cluster Coefficient & .198 & -.004 & & -- \\
\hline
\end{tabular}

Significant effects are highlighted in bold. 


\section{Table 3}

LMER analysis of accuracy for Disambiguation trials

\begin{tabular}{lllll}
\hline & Estimate & Std. Error & t-value & p-value \\
(Intercept) & -0.136 & 0.12 & -1.18 & .29 \\
Vocabulary size & -0.009 & 0.04 & -0.23 & .82 \\
Semantic category density & -0.063 & 0.04 & -1.61 & .11 \\
Global Cluster Coefficient & 0.005 & 0.09 & 0.06 & .95 \\
Voc. x Sem. Category Density & -0.003 & 0.04 & -0.09 & .93 \\
Voc. x GCC & 0.020 & 0.05 & -0.38 & .70 \\
Correlation of fixed effects & & & & \\
\hline & Intercept & 2 & & 3 \\
1. Vocabulary size & .065 & -.010 & & .488 \\
2. Semantic category density & $<.001$ & -- & & -.005 \\
3. Global Cluster Coefficient & .153 & -.005 & & -- \\
\hline
\end{tabular}


Table 4.

LMER analysis of accuracy for Retention trials

\begin{tabular}{lllll}
\hline & Estimate & Std. Error & t-value & p-value \\
(Intercept) & 0.057 & 0.13 & 0.45 & .67 \\
Vocabulary size & 0.023 & 0.05 & 0.50 & .62 \\
Semantic category density & 0.013 & 0.04 & 0.30 & .77 \\
Global Cluster Coefficient & -0.069 & 0.09 & -0.74 & .46 \\
Voc. x Sem. Category Density & 0.011 & 0.04 & 0.28 & .78 \\
Voc. x GCC & -0.066 & 0.06 & -1.19 & .24 \\
Correlation of fixed effects & & & & \\
\hline & Intercept & 2 & & 3 \\
1. Vocabulary size & .062 & -.005 & & .510 \\
2. Semantic category density & $<.001$ & -- & & -.006 \\
3. Global Cluster Coefficient & .145 & -.006 & & -- \\
\hline
\end{tabular}


Table 5.

Time windows where looks to Target significantly exceed Distractor during trial window starting at onset of spoken label (0 ms) to $4000 \mathrm{~ms}$ after label onset.

\begin{tabular}{|c|c|c|c|c|c|c|}
\hline & \multicolumn{3}{|c|}{ Semantic Category Density } & \multicolumn{3}{|c|}{ Global Clustering Coefficient } \\
\hline & Time window & Cluster T & $P$ value & Time window & Cluster T & $P$ value \\
\hline \multicolumn{7}{|c|}{ Familiar Trials } \\
\hline High & $100-2500 \mathrm{~ms}$ & 150.7 & $<.0001$ & $200-4000 \mathrm{~ms}$ & 257.2 & $<.0001$ \\
\hline Low & $200-3550 \mathrm{~ms}$ & 283.7 & $<.0001$ & $50-3500 \mathrm{~ms}$ & 264.7 & $<.0001$ \\
\hline \multicolumn{7}{|c|}{ Disambiguation Trials } \\
\hline High & None & -- & -- & None & -- & -- \\
\hline Low & None & -- & -- & None & -- & -- \\
\hline \multicolumn{7}{|c|}{ Retention Trials } \\
\hline High & $1800-2450 \mathrm{~ms}$ & 44.9 & .008 & None & -- & -- \\
\hline Low & None & -- & -- & None & -- & -- \\
\hline
\end{tabular}


Table 6.

Time windows where fixations vary by Semantic Category Density and Global Clustering Coefficient by experimental condition. Negative cluster values indicate Low > High, and Positive cluster values indicate High $<$ Low.

\begin{tabular}{|c|c|c|c|c|c|c|}
\hline & \multicolumn{3}{|c|}{ Semantic Category Density } & \multicolumn{3}{|c|}{ Global Clustering Coefficient } \\
\hline & Time window & Cluster T & $P$ value & Time window & Cluster T & $P$ value \\
\hline Familiar Trials & $1300-1450 \mathrm{~ms}$ & -9.61 & .001 & -- & -- & -- \\
\hline Disambiguation Trials & -- & -- & -- & -- & -- & -- \\
\hline Retention Trials & $2100-2300 \mathrm{~ms}$ & 13.19 & .001 & -- & -- & -- \\
\hline
\end{tabular}

